\title{
Estimation of Motion Vector Parameters using Modified Diamond Search
}

\author{
T.Swati \\ Assistant professor, \\ I. N.P.College of Engg \& \\ Technology \\ Kurnool,A.P,India
}

\author{
M.V.R.Vittal \\ Senior Assistant Professor, \\ G.P.R.Engg.College, \\ Kurnool,A.P,India
}

\begin{abstract}
A fast block motion estimation algorithm is proposed using modified diamond search patterns. This algorithm utilizes the directions and magnitudes of motion vectors between interblocks and uses a smaller number of search points than conventional diamond search patterns. Simulation results show that the proposed method significantly improves computational speed over other fast motion estimation algorithms without degradation of distortion.
\end{abstract}

\section{General Terms}

Search Techniques, signal processing, Algorithms

\section{Keywords}

Motion Vector Distribution (MVD), Modified Diamond Search (MDS), Block Matching Motion Estimation (BMME), Block Matching Algorithms (BMAs)

\section{INTRODUCTION}

Many video coding standards use block matching motion estimation (BMME) to reduce temporal redundancy between successive frames. However, the full search BMME requires a heavy computational burden. To speed up the motion estimation process, many fast block matching algorithms (BMAs) have been developed using different search patterns, such as square-shape, diamond-shape, and hexagon-shape by exploiting the motion vector distribution characteristics.

All fast BMAs are based on the assumption that the distortion of the block matching increases monotonically away from the global minimum distortion. The new three-step search (NTSS) employs a halfway-stop technique of the three-step search (TSS) using sparse square-shaped patterns, and results in accelerated motion estimation in stationary blocks. The diamond search (DS) algorithm and the cross diamond search (CDS) algorithm utilize centre-biased motion vector distribution (MVD) characteristics in real-life sequences.

DS and CDS algorithms are effective in cases of small motion, since their search patterns are based on the centre-biased MVD with a radius of 2 peels and use fewer search points with similar distortion performance compared to NTSS and TSS. In this Letter, we propose a faster and less distorted BMA which utilizes

Modified diamond patterns based on the motion correlation between neighbor blocks.

\section{MODIFIED DIAMOND SEARCH PATTERNS}

Most blocks in real-life image sequences have highly correlated motion characteristics between spatial and or temporal neighbor blocks, i.e. the current motion vector can be predicted from the neighbor blocks in a spatial and or temporal sense.

Hsieh and Lu introduced a halfway-stop technique that thresholds the motion compensation error with priority order between neighbor blocks. However, this motion estimation technique may not find the global minimum distortion or sudden changes of motion vectors along object boundaries, because the search regions are limited around the point predicted by motion vectors of neighbor blocks. In this Letter we propose the use of modified diamond search this algorithms is very popular for motion estimation and in fact it is used for motion estimation by MPEGTool. The basic idea behind this algorithm is to divide the search window into a number of regions and do a full search only in one of these regions. It may be described as:

Step 1: An initial step size is picked. Thirteen search points are selected surrounding a selected midpoint, in the pattern of a diamond, as shown in "(fig .1)" with the selected step size.

Step 2: A search is performed in the selected points and the point with minimum mod value is picked. Making this point as centre another nine points are selected in the diamond pattern. as shown in "(fig . 2)"

Step 3: A search is performed in the selected points and the point with minimum mod value is picked. If the point with minimum mod value is the midpoint then a diamond pattern of five search points is selected making the minimum mod value point as the centre.

Step 4: If the selected midpoint in the step 3 is not the midpoint then another diamond pattern with nine search points is selected around this point. This procedure is repeated until the minimum midpoint occurred is the midpoint

In the modified diamond search pattern with fewer search points by adapting inter block motion correlations. Three neighbor blocks, the left block and the upper block of a current block in a current frame and the block of the same pixel position in the previous frame, are used without the priority order between neighbor blocks to increase the likelihood of finding the global minimum distortion and sudden changes of motion vectors. 


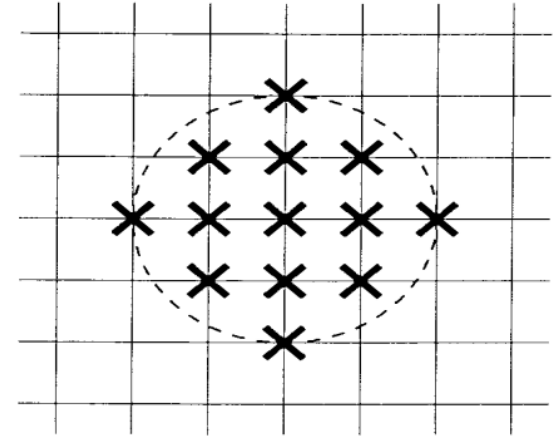

Fig. 1 Thirteen point pattern for diamond search
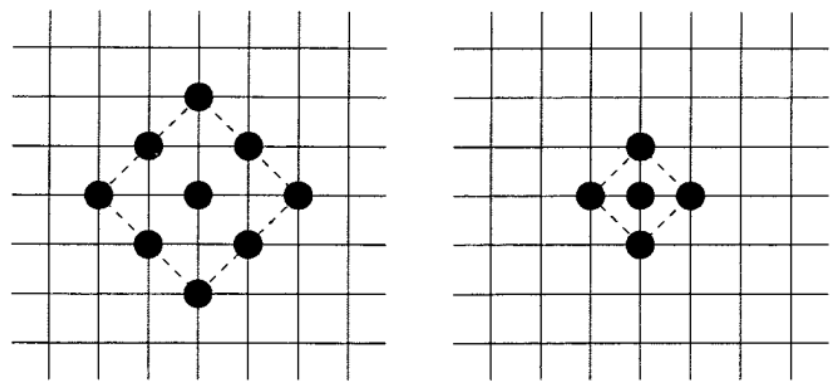

Fig. 2 Nine and five point pattern for diamond search

As shown in "(Fig. 3)", different search patterns are employed according to the motion vectors of neighbor blocks.

These patterns are based on the MVD found through experimentations, most image sequences have centre-biased motion vectors located in the central 5_5 area, which account for more than $80 \%$ of all motion vectors.

If all the motion vectors in the neighbor blocks have a distance of less than 3 peel $(\mathrm{p}<3)$, the proposed method uses the small sparse diamond (SSD) pattern "(Fig. 3a)". Otherwise, an initial search pattern is determined by motion directions predicted from those of neighbor blocks. If the neighbor blocks have the same motion directions within $90_{-}$, one of the quarter sparse diamond (QSD) patterns, QSD1, QSD2, QSD3, and QSD4, is selected based on their motion directions "(Fig. 3b)". If they are within 180_, one of the half sparse diamond (HSD) patterns "(Fig. 3c)", HSD1, HSD2, HSD3, and HSD4, is selected as the initial search pattern. In all other cases, HSD1 is used. Note that these QSD or HSD patterns are used for only the initial search pattern.

Modified diamond search (MDS) algorithm: The MDS patterns are based on the motion correlation characteristics of real-life image sequences. The MDS algorithm can be used for a faster BMA because they use fewer search points than DS and CDS patterns. The following is a summary of the MDS algorithm.

Step 1: If at least one of the displacements of the neighbor blocks is larger than 3, select one of the QSD or HSD patterns as the initial search pattern, depending on the directions of the neighbor motion vectors. Otherwise, set the centre point of the SSD pattern as the motion vector of $(0,0)$ and go to

Step 2. The point of the minimum block. Distortion measure (BDM) is found from the points in the initial search pattern, and this point is set as the centre point of the SSD pattern. Goto step 2. Step 2: Find the minimum distortion point using the centre point of the SSD pattern determined in step 1. If it is at the centre of the

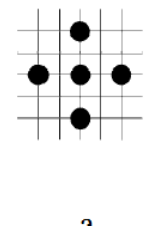

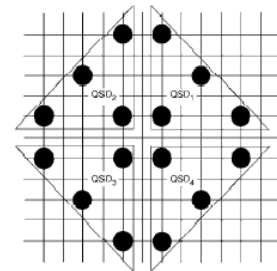

$\mathrm{b}$

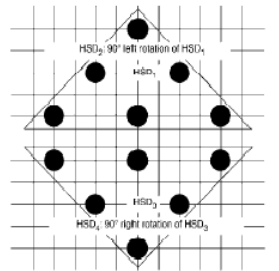

Fig. 3 Modified diamond search patterns exploiting motion correlation

a SSD pattern used as initial search pattern when $p<3$

b QSD patterns when $\mathbf{p} \_3$ and motion directions within 90 c HSD patterns when $p_{-} 3$ and motion directions within 180_, and in all other Cases

SSD pattern, go to step 3. Otherwise, repeat step 2 using the SSD pattern centered at the newly found minimum distortion point within the search range.

Step 3: Find the sets of two points with the smallest BDM from the outside points of the SSD pattern (denoted as or in "(Fig. 4a and b)". Those two points and the centre point of the SSD pattern define the boundaries of the candidates of final motion vectors. Identify a new BDM point from among these candidates, which is the final motion vector.

"(Fig. 4)" shows two examples of the motion estimation steps when the initial search patterns are different and the final motion vectors are the same as $(0,0)$. The circle, represents the minimum BDM point found in step 3. Note that the number of search points (NSP) in "(Figs. 4a and b)" is 8 and 13, respectively.

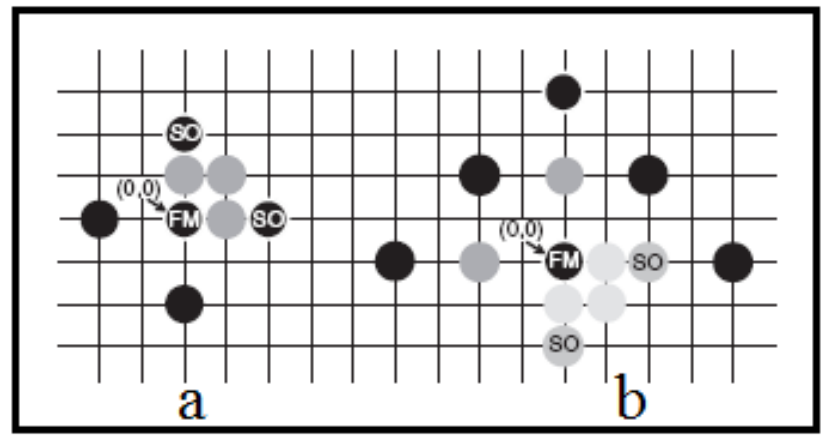

Fig. 4 Examples of proposed motion estimation algorithm a When $p<3$ in neighbor blocks

b When $p<3$ in neighbor blocks and motion directions within 180 


\section{BLOCK DIAGRAM}

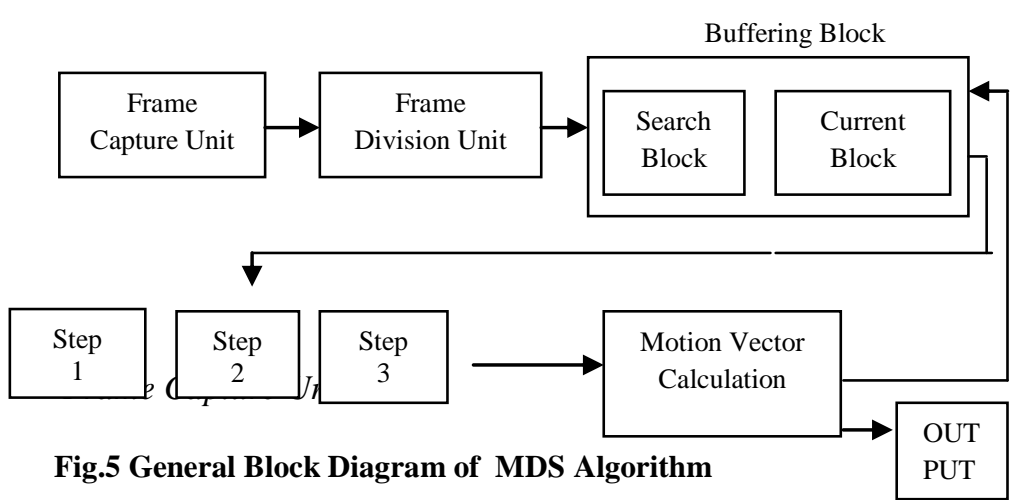

\subsection{Frame Capture Unit}

Frame capture unit captures the digital video formed of the frames and divides them into frames at a frequency of 30 frames/second. The frame capture unit supplies the captured frames to the frame division unit.

\subsection{Frame Division Unit}

Frame capture unit captures the digital video formed of the frames and divides them into frames at a frequency of 30frames/second. The frame capture unit supplies the captured frames to the frame division unit.

\subsection{Frame Division Unit}

Frame division unit collects the frame from the frame capture unit and divides them into blocks. Block motion estimation technique picks up a block from the current frame and compares it with the block in the previous frame. The frame division unit divides the current frame i.e. the frame to be estimated, into $16 \times 16$ blocks. The previous frame, which is taken as the reference, is divided into $32 \times 32$ blocks.

\subsection{Buffering Block}

The block from the current frame which is to be compared is placed into the current block and the block with which it is to be compared is placed into the search block. The address positions of the current and search blocks are always updated. When the first comparison is completed the position of the current block is to be moved by a length of 8pixels in all the possible ways. The position of the search block remains constant for the first complete comparison. A complete comparison is completed when the $16 \times 16$ current block is compared with the $32 \times 32$ search frame in all the possible ways as shown in the following "(figure 6)".
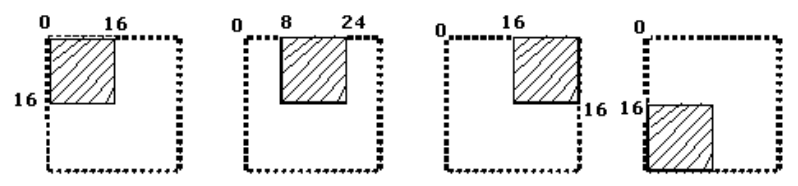

Fig .6 Current block on search block in all possible positions

\subsection{Algorithmic Block}

Modified diamond search block consists of three independent blocks each indicating one individual step in the diamond search algorithm.
The first step deals with the thirteen point diamond search and calculation of the block difference at that particular position. The second step is the nine point diamond search which is done making the minimum mod value in the thirteen point search as the midpoint and forming the nine point diamond around it. In the case of second search if the minimum mod value point is not the centre, then the nine point search is repeated, with the obtained minimum mod value point as the centre, until the point is obtained at the centre. The third step is performed with five search points and the centre being the centre of the nine point search.

\subsection{Motion Vector Block}

The $64 \times 2$ motion vectors are calculated from the obtained block difference and these are used to estimate the motion between the frames and thus to estimate the second frame using the first frame and the difference.

\subsection{Experimental Results}

The proposed MDS algorithm is simulated and the results were compared to those of FS, TSS, DS, CDS algorithms in terms of NSP, mean absolute difference (MAD) as the BDM, and the product of NSP and MAD "(Table 1)". All simulations were carried out with QCIF or CIF MPEG image sequences, block sizes of

16 , and search window sizes of _8. When there were small motions in the image sequences, such as those in the QCIF 'Miss America', MDS was able to achieve a similar performance as the next best algorithm, CDS, but about $15 \%$ faster. For slightly higher degrees of motion in QCIF sequences, such as 'Table Tennis', MDS was able to increase the speed by about $19 \%$. For CIF sequences with larger motions, MDS achieved 45\% ('Akiyo with Crowd') and 75\% ('Flower Garden') speed improvement with even better distortion performance. A smaller product of NSP and MAD can be considered as the figure-of-merit (FOM) for motion estimation algorithms. The MDS algorithm always resulted in a better FOM for all of the sequences, with up to $80 \%$ improvement ('Flower Garden') over CDS.

Table 1: Performance comparisons with other BMAs

\begin{tabular}{|l|l|l|l|l|l|l|l|}
\hline BMA & NSP & MAD & NSP *MAD & NSP & MAD & NSP * MAD \\
\hline & \multicolumn{6}{|l|}{ Miss America(QCIF) } & \multicolumn{2}{|c|}{ Table Tennis(QCIF) } \\
\hline FS & 289.0 & 1.775 & 339.586 & 289.0 & 1.84 & 531.625 \\
& & & & & & \\
\hline TSS & 25.00 & 1.177 & 29.425 & 24.96 & 2.032 & 50.734 \\
\hline DS & 13.33 & 1.176 & 15.678 & 13.71 & 1.84 & 25.221 \\
\hline CDS & 9.48 & 1.176 & 11.149 & 10.11 & 1.840 & 18.998 \\
\hline MDS & 8.23 & 1.178 & 9.693 & 8.45 & 1.889 & 15.963 \\
\hline
\end{tabular}




\section{CONCLUSION}

A fast BMA using MDS patterns is proposed by exploiting motion correlations in real-life image sequences. The experimental results show that this MDS algorithm can achieve faster motion estimation speeds with similar or even better distortions. The MDS algorithm outperforms other fast BMAs, and hence, it can be applied to various video applications for high speed motion estimation.

\section{REFERENCES}

[1] Li, R., Zeng, B., and Liou, M.L.: 'A new three-step search algorithm for block motion estimation', IEEE Trans. Circuits Syst. Video Technol., 1994, 4, pp. 438442.

[2] Shan, Z., and Ma, K.: 'A new diamond search algorithm for fast Block matching motion estimation', IEEE Trans. Image processes, 2000, 6, pp. 313-317.
[3] Cheung, C., and Po, L.: 'A novel cross-diamond search algorithm for fast block motion estimation', IEEE Trans. Circuits Syst. Video Technol.,2003, 12, pp. $1168-1177$

[4] Hsieh, C.H., and Lu, P.C.: 'Motion estimation using inter block correlation'. Proc. Int. Symp. On Circuits and Systems, May 1990, pp. 995-998

[5] Ding Gui-guang, guo Bao-long, "Motion Vector Estimation using Line Square Search Block Matching Algorithm for Video Sequence" EURASIP" journal on Applied Signal processing.Vol11,pp.1750-1756,2004.

[6] 6.Fukunga S, Nakaya Y,Son $\mathrm{S} \mathrm{H}$, Nagumo T, "MPeG-4 video verification model version [4.0[j]".in: ISO/IEC JTC/SC29/WG11 MPEG99/N2932, Victoria,Austalia,Oct. 1999 